\title{
Allele Frequency of 15 Short Tandem Repeats (Strs) in a Buganda Population (Central Uganda): Forensic Utility and Parentage Testing Jane Nabwowe ${ }^{1 *}$, Musa Kirya1, Emmanuel Okello² and Ann Nanteza² \\ ${ }^{1}$ Directorate of Government Analytical Laboratory, Uganda \\ ${ }^{2}$ Makerere University, Uganda
}

\begin{abstract}
The allele frequency of 15 Short tandem repeat (STRs) loci of the Buganda population-Central Uganda was determined in 221 unrelated individuals from the randomly selected 10 districts of the Buganda region. The DNA samples were extracted following Chelex extraction protocol, amplified using the AmpFISTR ${ }^{\circledR}$ Identifiler Plus ${ }^{\text {TM }}$ PCR amplification kit, and separated using capillary electrophoresis on an ABI 3130xI Genetic Analyzer. All loci were in Hardy-Weinberg equilibrium expectations and the Buganda population is heterozygous. When combined, combined power of exclusion (CPE), combined match probability (CMP) and combined power of discrimination (CPD) were $0.99981,1$ in $2.471 \times 10^{17}$ and 0.999999998 respectively an indication that the loci are highly informative, polymorphic and discriminative useful in paternity and forensic testing in the Buganda population.
\end{abstract}

Keywords: Short tandem repeats; Allele frequency; Buganda population

\section{Introduction}

Autosomal STR markers were first described as effective tools for human identity testing in the early 1990s [1] and have become the common currency of data exchange for human identity testing both in forensic casework and paternity testing [2]. Population databases allow for estimations of how rare or common a DNA profile may be in a particular population [3]. One population for which Short Tandem Repeats (STR) allele frequencies are not available is the Baganda tribe of Uganda. The aim of this work was to generate a Buganda population based 15 STR allele frequency data. We genotyped by multiplex PCR system using AmpF/STR ${ }^{\circledR}$ Identifiler ${ }^{\mathrm{TM}}$ Plus PCR Amplification Kit that co-amplifies the 13 Combined DNA Index System (CODIS) STR loci plus additional two tetrameric markers; D2S1338 and D19S433, as well as the amelogenin locus for gender identification. In this study we present the allele frequencies and statistical data of forensic importance in the Buganda population.

\section{Materials and Methods}

Ethical clearance was sought from the School of Biomedical Sciences Institutional Review Committee (IRC) College of Health Sciences of Makerere University, (Research file reference: SBS105) and Uganda National Council of Science and Technology (Research file reference: HS 1422). Blood samples were obtained from 221 unrelated individuals in the 10 randomly selected districts of the BugandaCentral Uganda who had previously consented. DNA was extracted following Chelex Extraction protocol [4] and amplified using the AmpFISTR $^{\circledR}$ Identifiler Plus ${ }^{\mathrm{TM}}$ PCR amplification kit following the manufacturer's user manual. Separation of the amplicons was done by capillary electrophoresis on an ABI 3130xl Genetic Analyzer using HiDi Formamide to fragment the bases and Gene Scan $500 \mathrm{Liz}$ to size the fragmented bases following Applied Biosystems Instruction Manual (2006): Starting Electrophoresis. The resulting STRs were genotyped using the GeneMapper ${ }^{\circledR}$ ID software V3.2. Allele frequency determination, Exact test probability for Hardy-Weinberg equilibrium (p-value), observed heterozygosity (Ho), expected heterozygosity $(\mathrm{He})$ and inbreeding coefficient expressed as Wright's fixation index $\left(\mathrm{F}_{\mathrm{is}}\right)$ were computed using GenePop v 4.1.3 software. Forensic and paternity efficiency parameters were computed using Promega Powerstat v12 excel spreed sheet.

\section{Results and Discussion}

The allele frequency of the 15 STRs generated of the Buganda population (Table 1) was below $50 \%$ at all loci as shown by the predominant alleles at each loci reflecting the usefulness and validity of these loci in calculating paternity indices and discriminating individuals [5]. All samples were successfully amplified and genotyped as shown by electropherogram images of the positive control; a quality control measure in genotyping process and selected samples of the Buganda population at the most heterozygous, polymorphic, and discriminating loci namely D21S11, D2S1338, D19S433 and FGA loci (Figure 1). All loci were in Hardy-Weinberg equilibrium (p-values $>0.05$ ) with the observed heterozygosity very close to the expected heterozygosity. High levels of observed heterozygosity ranging from $69.23 \%$ (D7S820) to $87.78 \%$ (D21S11) is an indication that the Buganda population has a high level of genetic variation and this could be successfully utilized in discriminating between individuals [6]. Both the heterozygosity and Fis values indicate that Buganda population is a heterozygous population with limited genetic inbreeding if any [7] and this is attributed to cultural practice of intermarriages among different clans and prohibition of marriages within the same clan.

All loci were highly polymorphic with polymorphic information content (PIC) values ranging from 0.67 at D13S317 to 0.89 at D2S1338 all above 0.5 indicating good informativeness of all the tested STR markers and useful for identification purposes [6]. The power of discrimination (PD) across all tested loci was above $80 \%$ (from 0.875 at $\mathrm{D} 13 \mathrm{~S} 317$ to 0.978 at D2S1338) an indication that all loci are highly discriminating, that is, an innocent person will be excluded as the donor of an evidence unknown sample [6]. The most informative markers were

*Corresponding author: Ms. Jane Nabwowe, Directorate of Government Analytical Laboratory, Uganda, Tel: +256712553836; E-mail: jnabwowe@yahoo.com

Received November 13, 2013; Accepted January 09, 2014; Published January 13, 2014

Citation: Nabwowe J, Kirya M, Okello E, Nanteza A (2014) Allele Frequency of 15 Short Tandem Repeats (Strs) in a Buganda Population (Central Uganda): Forensic Utility and Parentage Testing. J Forensic Res 5: 216. doi:10.4172/2157 7145.1000216

Copyright: (c) 2014 Nabwowe J, et al. This is an open-access article distributed under the terms of the Creative Commons Attribution License, which permits unrestricted use, distribution, and reproduction in any medium, provided the original author and source are credited. 
Citation: Nabwowe J, Kirya M, Okello E, Nanteza A (2014) Allele Frequency of 15 Short Tandem Repeats (Strs) in a Buganda Population (Central Uganda): Forensic Utility and Parentage Testing. J Forensic Res 5: 216. doi:10.4172/2157-7145.1000216

Page 2 of 4

\begin{tabular}{|c|c|c|c|c|c|c|c|c|c|c|c|c|c|c|c|}
\hline Allele & D8S1179 & D21S11 & D7S820 & CSFIPO & D3S1358 & TH01 & D13S317 & D16S539 & D2S1338 & D19S433 & vWA & TPOX & D18S51 & D5S818 & FGA \\
\hline 6 & _ & _ & & 0.002 & _ & 0.17 & _ & _ & _ & _ & _ & 0.081 & _ & _ & \\
\hline 7 & & - & $\overline{0} .011$ & 0.045 & - & 0.396 & & & & - & & 0.011 & - & & \\
\hline 8 & $\overline{0.002}$ & - & 0.195 & 0.027 & - & 0.222 & $\overline{0} .02$ & $\overline{0.045}$ & - & - & - & 0.292 & - & $\overline{0.059}$ & - \\
\hline 9 & _ & - & 0.102 & 0.138 & - & 0.161 & 0.016 & 0.224 & 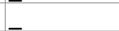 & 0.009 & 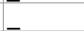 & 0.271 & - & 0.014 & 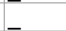 \\
\hline 9.3 & & - & & & - & 0.038 & & & 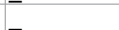 & & & & 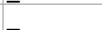 & & \\
\hline 10 & $\overline{0} .007$ & - & $\overline{0} .423$ & $\overline{0} .283$ & - & 0.009 & $\overline{0.032}$ & $\overline{0} .124$ & - & $\overline{0.014}$ & & $\overline{0} .077$ & & $\overline{0.066}$ & - \\
\hline 11 & 0.048 & - & 0.172 & 0.179 & - & 0.002 & 0.348 & 0.303 & - & 0.088 & $\overline{0} .007$ & 0.231 & 0.002 & 0.219 & - \\
\hline 11.2 & & & & & & & & & & 0.007 & & & & & - \\
\hline 12 & $\overline{0} .138$ & - & $\overline{0} .086$ & $\overline{0} .251$ & $\overline{0} .002$ & $\overline{0} .002$ & $\overline{0.351}$ & $\overline{0} .197$ & - & 0.113 & $\overline{0} .002$ & $\overline{0} .034$ & $\overline{0} .02$ & $\overline{0} .342$ & - \\
\hline 12.2 & & - & & & & & & & & 0.018 & & & & & \\
\hline 13 & $\overline{0} .138$ & - & $\overline{0} .011$ & $\overline{0} .068$ & $\overline{0} .002$ & - & $\overline{0} .186$ & $\overline{0.095}$ & $\overline{0} .002$ & 0.226 & $\overline{0} .025$ & $\overline{0} .002$ & $\overline{0} .061$ & $\overline{0.271}$ & - \\
\hline 13.2 & & & & & & & & & & 0.086 & & & 0.007 & & \\
\hline 14 & $\overline{0} .33$ & - & - & $\overline{0} .007$ & $\overline{0} .095$ & - & $\overline{0.045}$ & $\overline{0.011}$ & - & 0.238 & $\overline{0} .1$ & - & 0.057 & $\overline{0.025}$ & _ \\
\hline 14.2 & & - & - & - & & - & & & - & 0.038 & & - & 0.007 & & - \\
\hline 15 & $\overline{0} .256$ & - & - & - & $\overline{0} .324$ & - & $\overline{0} .002$ & - & - & 0.068 & $\overline{0} .215$ & - & 0.17 & $\overline{0.002}$ & 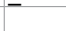 \\
\hline 15.2 & & - & - & - & & - & 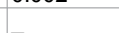 & - & - & 0.077 & & - & 0.007 & 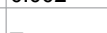 & - \\
\hline 16 & $\overline{0.066}$ & - & - & - & $\overline{0} .328$ & - & - & - & 0.07 & 0.007 & $\overline{0} .242$ & - & 0.21 & - & \\
\hline 16.2 & & - & - & - & & - & - & - & & 0.009 & & - & 0.007 & & \\
\hline 17 & $\overline{0} .014$ & - & - & - & $\overline{0} .204$ & - & - & - & $\overline{0} .127$ & 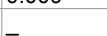 & $\overline{0} .186$ & - & 0.176 & $\overline{0} .002$ & $\overline{0} .002$ \\
\hline 18 & 0.002 & - & - & - & 0.045 & - & - & - & 0.095 & - & 0.143 & - & 0.136 & - & 0.005 \\
\hline 18.2 & _ & - & - & - & 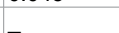 & - & - & - & & - & & - & 0.002 & - & \\
\hline 19 & - & - & - & - & - & - & - & - & $\overline{0} .145$ & & $\overline{0} .061$ & - & 0.068 & - & $\overline{0} .043$ \\
\hline 13.2 & & - & - & & & - & & & & $\overline{0} .086$ & & - & 0.007 & & \\
\hline 14 & $\overline{0} .33$ & - & - & $\overline{0.007}$ & $\overline{0} .095$ & - & $\overline{0.045}$ & $\overline{0.011}$ & - & 0.238 & $\overline{0} .1$ & - & 0.057 & $\overline{0.025}$ & - \\
\hline 14.2 & & - & & & & & & & & 0.038 & & & 0.007 & & \\
\hline 15 & $\overline{0} .256$ & - & - & - & $\overline{0} .324$ & - & $\overline{0} .002$ & - & - & 0.068 & $\overline{0} .215$ & - & 0.17 & $\overline{0} .002$ & - \\
\hline 15.2 & & & & - & & & & - & & 0.077 & & - & 0.007 & 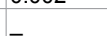 & - \\
\hline 16 & $\overline{0} .066$ & - & - & - & $\overline{0} .328$ & 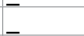 & - & - & $\overline{0} .07$ & 0.007 & $\overline{0} .242$ & - & 0.21 & - & - \\
\hline 16.2 & & - & - & - & & - & - & - & & 0.009 & & - & 0.007 & & \\
\hline 17 & $\overline{0} .014$ & - & - & - & $\overline{0} .204$ & - & - & - & $\overline{0} .127$ & & $\overline{0} .186$ & - & 0.176 & $\overline{0.002}$ & $\overline{0} .002$ \\
\hline 18 & 0.002 & - & - & - & 0.045 & - & - & - & 0.095 & - & 0.143 & - & 0.136 & 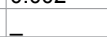 & 0.005 \\
\hline 18.2 & 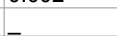 & - & - & - & _ & - & - & - & & - & & - & 0.002 & - & \\
\hline 19 & - & - & - & - & - & - & - & - & $\overline{0} .145$ & - & $\overline{0} .061$ & - & 0.068 & - & $\overline{0} .043$ \\
\hline 19.2 & - & - & - & - & - & - & - & - & & - & & - & & - & 0.002 \\
\hline 20 & - & - & - & - & - & - & - & - & $\overline{0} .063$ & & $\overline{0} .02$ & - & $\overline{0} .041$ & - & 0.036 \\
\hline 21 & - & - & - & - & - & - & - & - & 0.133 & $\overline{0} .002$ & _ & - & 0.02 & - & 0.077 \\
\hline 22 & - & - & - & - & _ & - & - & - & 0.113 & 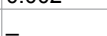 & - & - & 0.005 & - & 0.231 \\
\hline 23 & - & - & - & - & - & 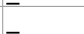 & - & - & 0.095 & 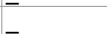 & - & - & 0.002 & - & 0.176 \\
\hline 24 & - & & - & - & - & - & - & - & 0.072 & - & - & - & .00 & - & 0.156 \\
\hline 24.2 & - & $\overline{0} .002$ & - & - & - & - & - & - & & - & - & - & - & - & \\
\hline 25 & - & _ & - & - & - & - & - & - & $\overline{0} .043$ & - & - & - & - & - & $\overline{0} .102$ \\
\hline 26 & - & & - & - & - & - & - & - & 0.025 & - & - & - & - & - & 0.057 \\
\hline 27 & - & $\overline{0.05}$ & & - & - & - & - & - & 0.016 & - & - & - & - & - & 0.052 \\
\hline 28 & - & 0.242 & - & - & - & - & - & - & 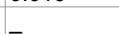 & - & - & - & & - & 0.02 \\
\hline 29 & - & 0.226 & - & - & - & - & - & - & - & - & - & - & $\overline{0} .002$ & - & 0.018 \\
\hline 29.2 & - & & & & - & - & - & - & - & - & - & - & _ & - & 0.002 \\
\hline 30 & - & $\overline{0} .174$ & - & - & - & - & - & - & - & - & - & - & - & - & 0.005 \\
\hline 30.2 & - & 0.007 & & - & - & - & - & - & - & - & - & - & - & - & 0.007 \\
\hline 31 & - & 0.093 & - & - & - & - & - & - & - & - & - & - & - & - & \\
\hline 31.2 & - & 0.034 & - & - & - & - & - & - & - & - & - & - & - & - & $\overline{0} .007$ \\
\hline 32 & - & 0.016 & - & - & - & - & $\begin{array}{l}- \\
-\end{array}$ & - & - & - & - & - & - & - & \\
\hline 32.2 & - & 0.061 & - & $\begin{array}{lll}- \\
-\end{array}$ & - & - & - & - & - & - & - & - & - & $\begin{array}{l}- \\
-\end{array}$ & $\overline{0} .002$ \\
\hline 33 & & 0.005 & & & - & - & - & - & - & - & - & - & - & $\begin{array}{l}- \\
-\end{array}$ & \\
\hline 33.2 & - & 0.027 & - & $\begin{array}{lll}- & \\
\end{array}$ & - & - & - & - & - & - & - & - & - & $\begin{array}{l}- \\
-\end{array}$ & - \\
\hline 34 & - & 0.016 & - & - & - & - & - & - & - & - & - & - & - & - & - \\
\hline 34.2 & - & 0.002 & - & - & - & - & - & - & - & - & - & - & - & - & - \\
\hline 35 & - & 0.029 & - & $\begin{array}{lll}- \\
-\end{array}$ & - & - & - & - & - & - & - & - & - & - & - \\
\hline 36 & - & 0.011 & & & - & - & - & - & - & - & - & - & - & - & - \\
\hline 37 & & 0.005 & & & & & & & & & & & & & \\
\hline$H(\exp )$ & $\overline{0} .782$ & 0.843 & $\overline{0} .737$ & $\overline{0} .800$ & $\overline{0} .737$ & $\overline{0} .800$ & $\overline{0} .719$ & $\overline{0} .794$ & $\overline{0} .899$ & $\overline{0} .854$ & $\overline{0} .828$ & $\overline{0} .776$ & $\overline{0} .865$ & $\overline{0} .755$ & $\overline{0} .867$ \\
\hline H (obs) & 0.719 & 0.878 & 0.692 & 0.819 & 0.747 & 0.783 & 0.729 & 0.760 & 0.864 & 0.824 & 0.805 & 0.742 & 0.842 & 0.697 & 0.864 \\
\hline Fis & 0.041 & -0.023 & 0.083 & -0.005 & -0.013 & -0.036 & -0.025 & 0.020 & 0.031 & 0.012 & 0.028 & 0.049 & 0.013 & 0.044 & 0.002 \\
\hline $\mathrm{P}$ & 0.093 & 0.128 & 0.183 & 0.850 & 0.250 & 0.543 & 0.691 & 0.210 & 0.351 & 0.379 & 0.325 & 0.066 & 0.545 & 0.720 & 0.790 \\
\hline MP & 0.079 & 0.050 & 0.105 & 0.075 & 0.120 & 0.114 & 0.125 & 0.074 & 0.022 & 0.040 & 0.054 & 0.091 & 0.035 & 0.095 & 0.033 \\
\hline PD & 0.921 & 0.95 & 0.895 & 0.925 & 0.88 & 0.886 & 0.875 & 0.926 & 0.978 & 0.96 & 0.946 & 0.909 & 0.965 & 0.905 & 0.967 \\
\hline PIC & 0.75 & 0.82 & 0.7 & 0.77 & 0.69 & 0.7 & 0.67 & 0.76 & 0.89 & 0.84 & 0.8 & 0.74 & 0.85 & 0.71 & 0.85 \\
\hline $\mathrm{PE}$ & 0.459 & 0.75 & 0.416 & 0.635 & 0.504 & 0.568 & 0.474 & 0.527 & 0.723 & 0.643 & 0.609 & 0.496 & 0.678 & 0.423 & 0.723 \\
\hline TPI & 1.78 & 4.09 & 1.63 & 2.76 & 1.97 & 2.3 & 1.84 & 2.08 & 3.68 & 2.83 & 2.57 & 1.94 & 3.16 & 1.65 & 3.68 \\
\hline
\end{tabular}

$H(\exp )$ : Expected heterozygosity

$\mathrm{H}$ (obs): Observed heterozygosity

Fis: Wright's fixation index

P: Exact test probability for Hardy-Weinberg equilibrium

MP: Matching Probability

DP: Power of Discrimination

PIC: Polymorphic Information Content

PE: Power of Exclusion

TPI: Typical Paternity Index

Table 1: Allele frequency and Forensic statistical parameters of 15 short tandem repeats loci (STRs) in a Buganda Population-Central Uganda ( $n=221 ; n$; number of individuals sampled). 
Citation: Nabwowe J, Kirya M, Okello E, Nanteza A (2014) Allele Frequency of 15 Short Tandem Repeats (Strs) in a Buganda Population (Central Uganda): Forensic Utility and Parentage Testing. J Forensic Res 5: 216. doi:10.4172/2157-7145.1000216

Page 3 of 4
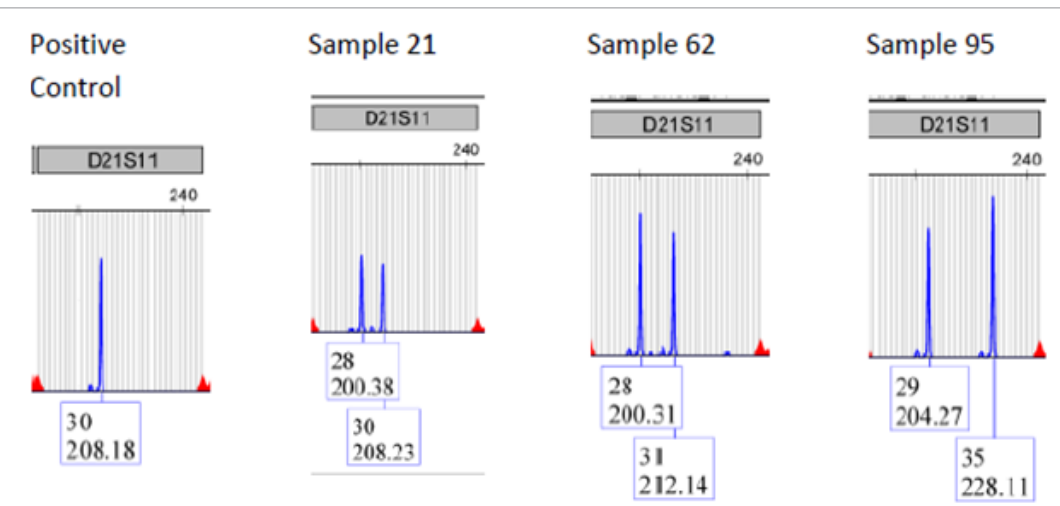

Sample 108
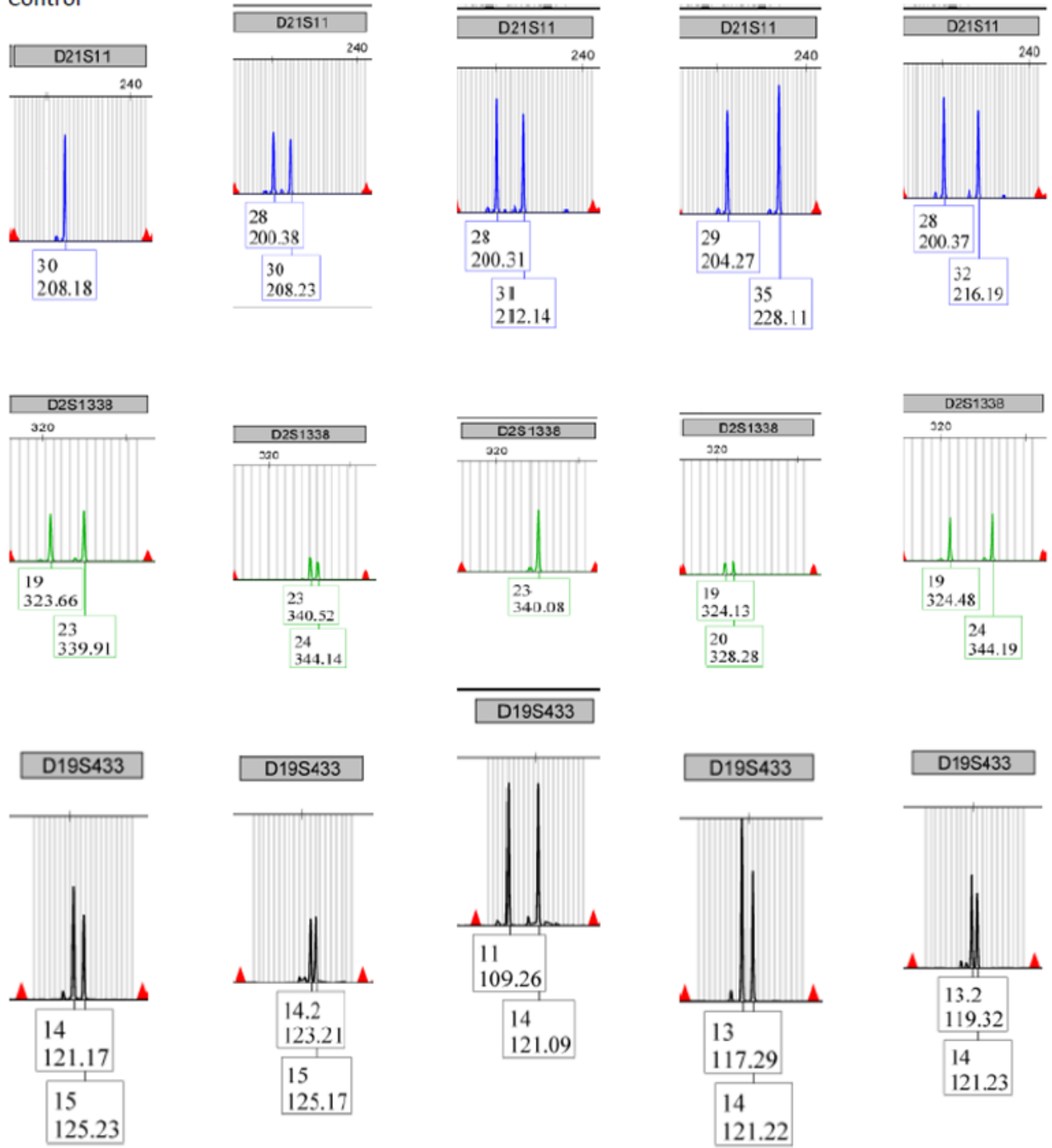

Positive

Control
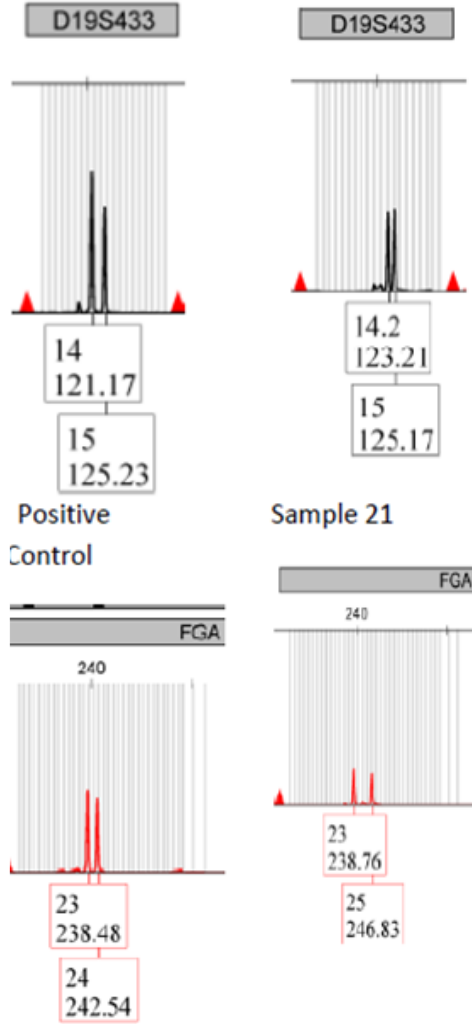

\section{D19S433}

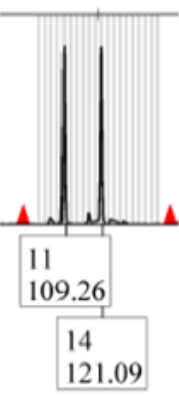

\section{D19S433}

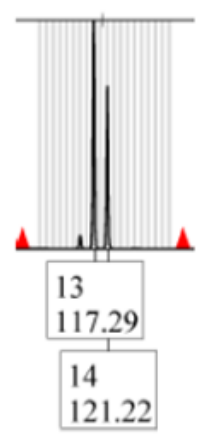

Sample 95

Sample 62

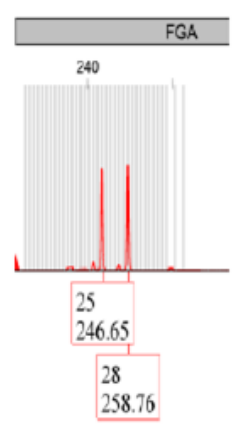

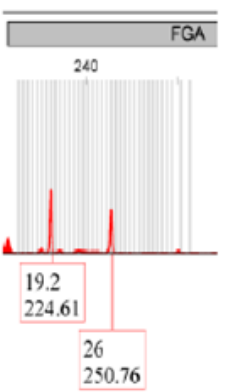

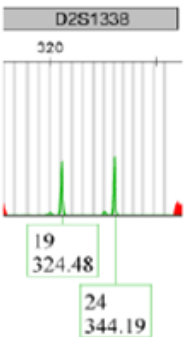

D19S433

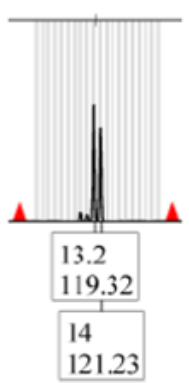

Sample 108

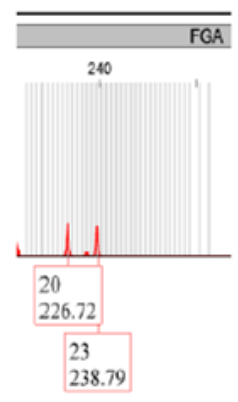

Figure 1: Electropherograms for the positive control and sample 21, sample 62, sample 95 and sample 108 of the Buganda population showing allele call and DNA size in base pairs (bp) at D21S11, D2S1338, D19S433 and FGA loci. The positive control; a quality control measure in genotyping process illustrates that amplification and genotyping were successful. These loci were highly heterozygous and polymorphic with high discriminating $(P D)$ values and power of exclusion (PE) values. Microvariant alleles were observed at some loci in the Buganda population as shown at D19S433 of sample 21 and FGA of sample 95. The DNA molecules are separated according to their respective sizes. So the smallest fragments are chronologically detected first and if two (or more) fragments have the same size, they are distinguished by the fluorescence color. The use of colors (blue, green, yellow and red) with different diffusivity included in the PCR multiplex, allow distinguishing the overlapping fragments (same size). 
Citation: Nabwowe J, Kirya M, Okello E, Nanteza A (2014) Allele Frequency of 15 Short Tandem Repeats (Strs) in a Buganda Population (Central Uganda): Forensic Utility and Parentage Testing. J Forensic Res 5: 216. doi:10.4172/2157-7145.1000216

Page 4 of 4

the most discriminating ones in the Buganda population as it was in the population of Island of Cres, Croatia [7]. The calculated combined match probability (CMP) of 1 in $2.471 \times 10^{17}$ and the combined power of discrimination (CPD) which was greater than 0.999999998 in the Buganda population will have an important role in the analysis of mixed DNA profiles such as in sexual assault cases as well as matching a control sample to the unknown sample [6].

The typical paternity index values at all loci were greater than 1 , indicative of relatedness $[8,9]$. High paternity exclusion (PE) values ranging from 0.416 (D7S820) to 0.750 (D21S11) were observed for the Buganda population which showed that the loci could powerfully exclude a falsely accused individual as a biological father to a child and the higher the PE value, the more non-fathers are excluded [9]. Combined, the 15 loci yielded a combined typical paternity index (CTPI) of 565,852.41 and a combined power of paternity exclusion (CPE) of 0.99981 (greater than 99\%) indicating a high power of exclusion [6].

\section{Conclusion}

The Buganda population is genetically diverse, all loci were very informative, highly polymorphic with high power of discrimination and exclusion. When combined, these loci are powerful genetic markers for forensic identification and paternity testing in the Buganda population.

\section{Acknowledgements}

The authors would like to thank the National DNA Laboratory; Directorate of
Government Analytical Laboratory, Ministry of Internal Affairs Kampala where the work was carried out and the laboratory staff for their careful review and thoughtful comments. We are grateful to Mr. Kutranov Stefan; Forensic Regional Account Manager (Promega) for providing us the software used in the analysis of this work and his timely technical advice.

\section{References}

1. Edwards A, Civitello A, Hammond HA, Caskey CT (1991) DNA typing and genetic mapping with trimeric and tetrameric tandem repeats. Am J Hum Genet 49: 746-756.

2. Ballantyne J (1997) Mass disaster genetics. Nat Genet 15: 329-331.

3. Ban JD (2001) Establishing a large DNA data bank using the PowerPlex 1.1 and 2.1 systems. Croat Med J 42: 256-259.

4. Walsh PS, Metzer DA, Higuch R (1991) Chelex 100 as a medium for simple extraction for simple extraction of DNA for PCR- Based typing from Forensic material. BioTechniques 10: 506-513.

5. Weir BS (1996) In: Genetics Data Analysis II. Sinauer Associates Inc. Sunderland, MA, USA.

6. Butler JM (2005) Forensic DNA Typing: Biology, Technology, and Genetics of STR Markers. (2ndedn), Elsevier Academic Press, Massachusetts, USA.

7. Nei M, Roychoudhury AK (1974) Sampling variances of heterozygosity and genetic distance. Genetics 76: 379-390.

8. Natalija N, Marjanovi D, Skaro V, Projic P, Lauc G, et al. (2009) Forensic Efficiency Parameters for the 15 STR Loci in the Population of the Island of Cres (Croatia) Coll. Antropol 33: 1319-1322.

9. Manamperi A, Hapuarachchi C, Gunawardene NS, Bandara A, Dayanath D et al. (2009) STR polymorphisms in Sri Lanka: evaluation of forensic utility in identification of individuals and parentage testing. Ceylon Med J 54: 85-89. 